\title{
Peroral endoscopic myotomy (POEM) in a 3-year-old girl with severe growth retardation, achalasia, and Down syndrome
}

Achalasia is a primary motility disorder with an annual incidence of 0.11 cases/ 100000 children [1]. In the field of natural orifice transluminal endoscopic surgery (NOTES), the peroral endoscopic myotomy (POEM) [2-3] is the only treatment to be performed routinely in humans. We report the case of a 3-year old female patient with Down syndrome who presented with severe growth retardation and weight at less than the $3 \mathrm{rd}$ percentile curve. Achalasia was suspected because of vomiting, recurrent cough, aspiration pneumonia, and growth retardation; it was confirmed by barium esophagogram, endoscopy, and esophageal manometry.

The first treatment was pneumatic balloon dilation (balloon diameter $20 \mathrm{~mm}$ ). Although the lower esophageal sphincter pressure improved after dilation, the patient continued to vomit daily. POEM was performed 20 days after balloon dilation. With the exception of the gastroscope, which had a diameter of $9.0 \mathrm{~mm}$ (GFXQ 260; Olympus, Tokyo, Japan), POEM was performed using the same equipment as that used in adult patients and followed the same steps ( $\bullet$ Fig. 1): distal attachment (MH-587, Olympus); triangle-tip knife (KD640L; Olympus) to dissect the submucosal layer and perform myotomy; coagulating forceps (Coagrasper, FD$411 \mathrm{QR}$; Olympus) for hemostasis; $\mathrm{CO}_{2}$ in- sufflator (UCR; Olympus), VIO-300 D electrogenerator (ERBE, Tübingen, Germany), and hemostatic clips (EZ-CLIP, HX-110QR; Olympus) to close the mucosal entry. The generator set-up was also the same as that used for adults: endocut Q-mode (effect 2) for mucosal entry, spray coagulation mode (effect 2; 50 watts) to create the submucosal tunnel and for myotomy, and soft coagulation (effect 5; 80 watts) for hemostasis. The operation time was 198 minutes. There were no intraoperative or postoperative complications. Upper digestive endoscopy and barium esophagogram confirmed smooth passage through the gastroesophageal junction, without leakage ( $\bullet$ Fig. 2 ).

At 1 year after the procedure, the patient was asymptomatic and a normal weight for her age.

To our knowledge, this is the first case of POEM in a 3-year-old child, and it demonstrates that POEM is feasible and successful in pediatric patients with achalasia.

Endoscopy_UCTN_Code_TTT_1AO_2AN

Competing interests: $\mathrm{H}$. Inoue has received research and educational grants from Olympus, Boston Scientific, and Ethicon Endosurgery on behalf of the Digestive Disease Center, Northern Yokohama Hospital.
R. Maselli' ${ }^{1}$, H. Inoue ${ }^{1}$, M. Misawa ${ }^{1}$, H. Ikeda ${ }^{1}$, T. Hosoya ${ }^{1}$, M. Onimaru ${ }^{1}$, A. Yoshida ${ }^{1}$, N. Eleftheriadis' ${ }^{1}$, K. Suzuki ${ }^{2}$, S. Kudo ${ }^{1}$

${ }^{1}$ Digestive Disease Center, Showa University Northern Yokohama Hospital, Tsuzuki-ku, Yokohama, Japan

2 Pediatric Center, Showa University Northern Yokohama Hospital, Tsuzuki-ku, Yokohama, Japan

\section{References}

1 Hussain SZ, Thomas $R$, Tolia $V$. A review of achalasia in 33 children. Dig Dis Sci 2002; 47: $2538-2543$

2 Inoue $H$, Minami H, Kobayashi Yet al. Peroral endoscopic myotomy (POEM) for esophageal achalasia. Endoscopy 2010; 42: $265-$ 271

3 Inoue $\mathrm{H}$, Tianle KM, Ikeda $\mathrm{H}$ et al. Peroral endoscopic myotomy for esophageal achalasia: technique, indication, and outcomes. Thorac Surg Clin 2011; 21: 519-525

\section{Bibliography}

Dol http://dx.doi.org/

10.1055/s-0032-1309924

Endoscopy 2012; 44: E285-E287

(c) Georg Thieme Verlag KG

Stuttgart · New York

ISSN 0013-726X

Corresponding author

\section{R. Maselli, MD}

Digestive Disease Center

Showa University Northern Yokohama Hospital

35-1 Chigasakichuo

Tsuzuki-ku

Yokohama, 224-8503

Japan

Fax: +81-45-949-7000

roberta.maselli.md@gmail.com

- Fig. 1 and 2 see following pages. 

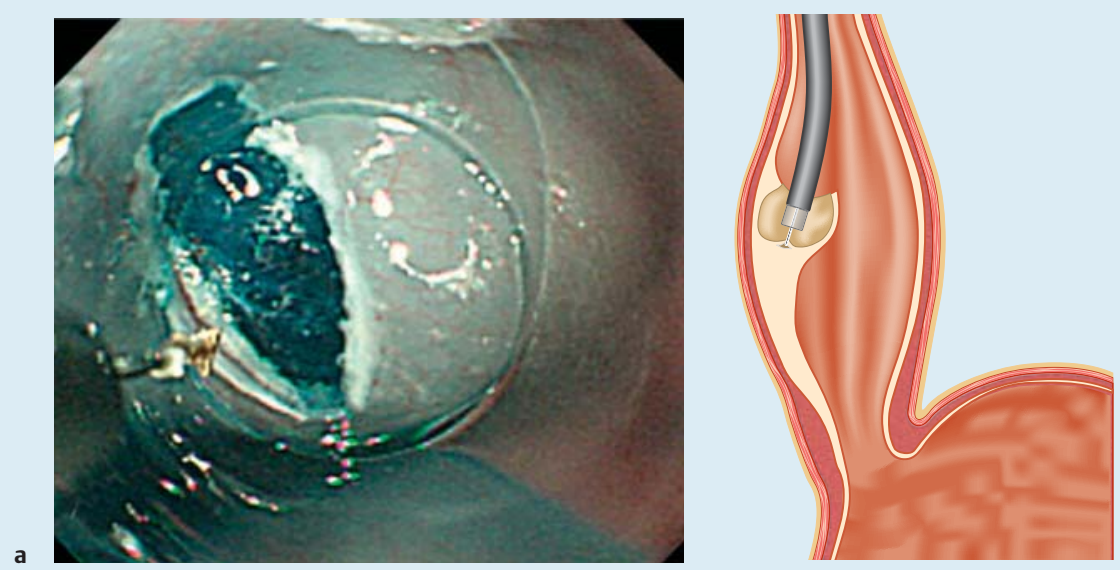

Fig. 1 Main steps of the peroral endoscopic myotomy procedure. Intraoperative images (left) and schematic explanation (right). a Entry to submucosal space. $\mathbf{b}$ Creation of a submucosal tunnel distal to the gastroesophageal junction. $\mathbf{c}$ Endoscopic myotomy (beginning $2 \mathrm{~cm}$ distal to the mucosal entry) of inner circular muscle. $\mathbf{d}$ Closure of the mucosal entry by hemostatic clips.
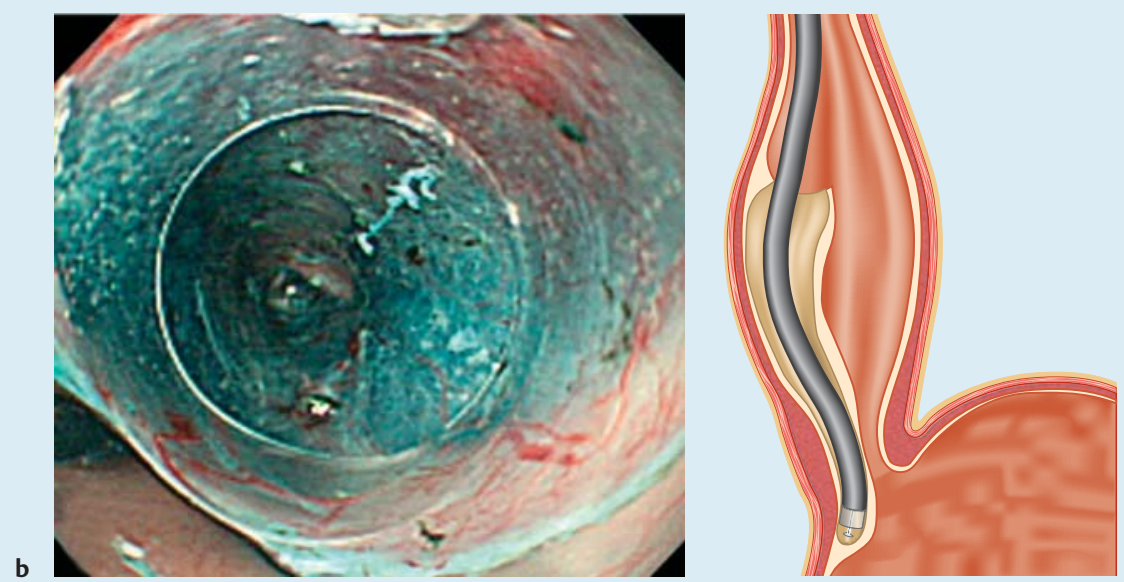

c
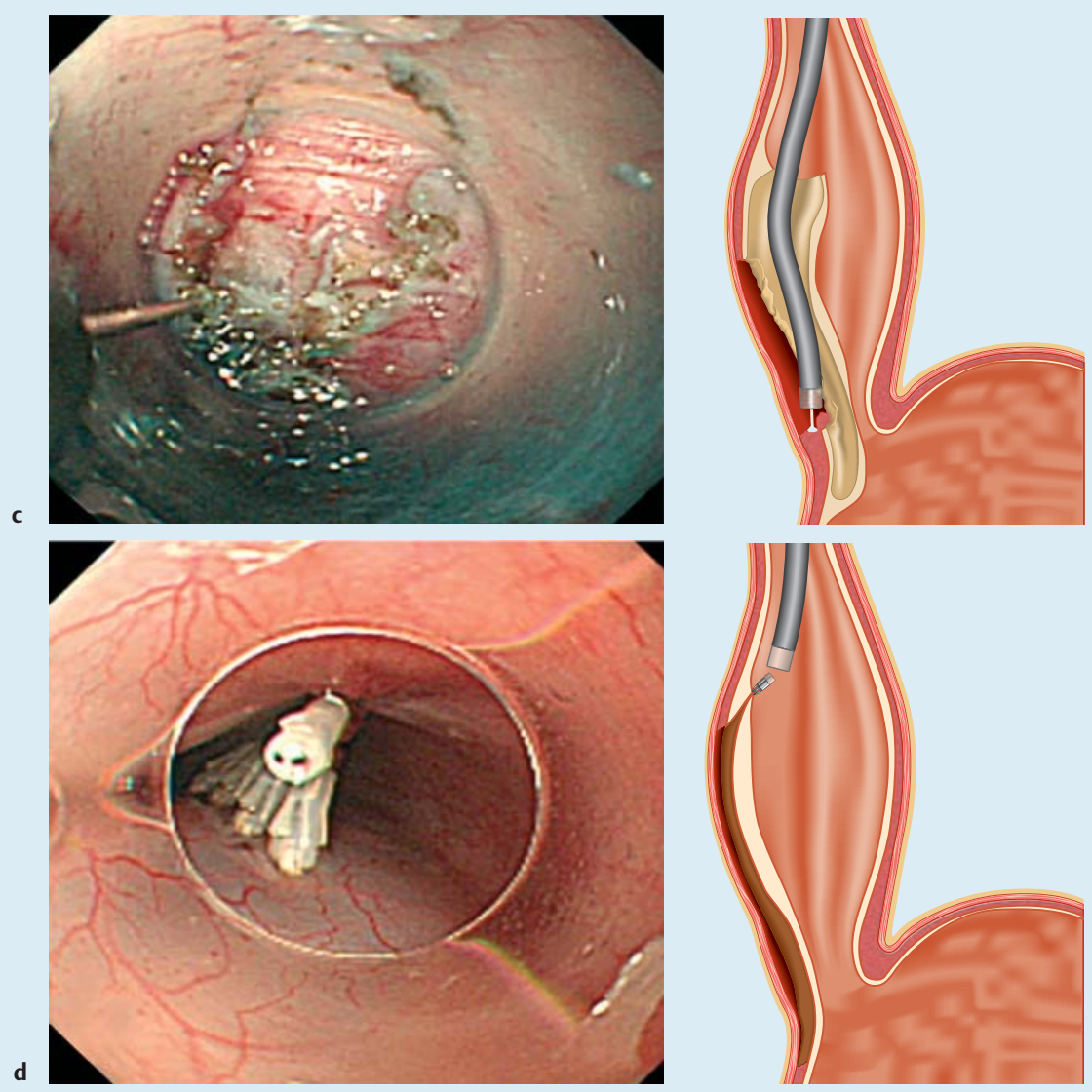


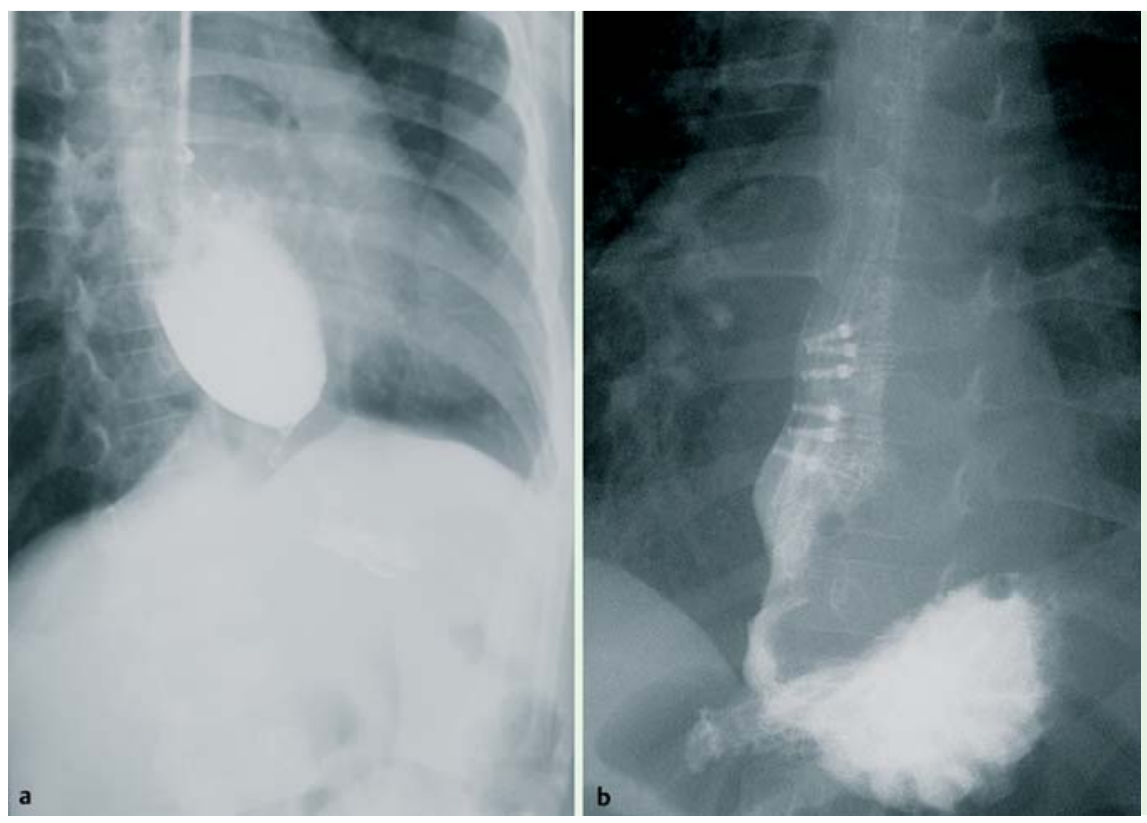

Fig.2 Barium esophagograms of the patient before (a) and after (b) peroral endoscopic myotomy. a Dilated esophagus with the classic "bird's beak" in the distal esophagus. $\mathbf{b}$ Normal barium passage from the distal esophagus to the stomach; the hemostatic clips can be seen in the mid esophagus. 